\title{
XRCCI and PCNA are loading platforms with distinct kinetic properties and different capacities to respond to multiple DNA lesions
}

\author{
Oliver Mortusewicz and Heinrich Leonhardt*
}

Address: Ludwig Maximilians University Munich, Department of Biology II, 82152 Planegg-Martinsried, Germany

Email: Oliver Mortusewicz - o.mortusewicz@lmu.de; Heinrich Leonhardt* - H.Leonhardt@lmu.de

* Corresponding author

Published: 19 September 2007

BMC Molecular Biology 2007, 8:8I doi:10.1 186/147|-2199-8-8I

This article is available from: http://www.biomedcentral.com/I47I-2199/8/8।

(c) 2007 Mortusewicz and Leonhardt; licensee BioMed Central Ltd.

This is an Open Access article distributed under the terms of the Creative Commons Attribution License (http://creativecommons.org/licenses/by/2.0), which permits unrestricted use, distribution, and reproduction in any medium, provided the original work is properly cited.

\begin{abstract}
Background: Genome integrity is constantly challenged and requires the coordinated recruitment of multiple enzyme activities to ensure efficient repair of DNA lesions. We investigated the dynamics of $\mathrm{XRCCI}$ and PCNA that act as molecular loading platforms and play a central role in this coordination.

Results: Local DNA damage was introduced by laser microirradation and the recruitment of fluorescent $\mathrm{XRCCI}$ and PCNA fusion proteins was monitored by live cell microscopy. We found an immediate and fast recruitment of $X R C C I$ preceding the slow and continuous recruitment of PCNA. Fluorescence bleaching experiments (FRAP and FLIP) revealed a stable association of PCNA with DNA repair sites, contrasting the high turnover of $\mathrm{XRCCl}$. When cells were repeatedly challenged with multiple DNA lesions we observed a gradual depletion of the nuclear pool of PCNA, while XRCCI dynamically redistributed even to lesions inflicted last.
\end{abstract}

Conclusion: These results show that PCNA and XRCCI have distinct kinetic properties with functional consequences for their capacity to respond to successive DNA damage events.

\section{Background}

Mammalian cells have to deal with a wide variety of different DNA lesions caused by cellular metabolites, replication errors, spontaneous disintegration and environmental influences. These lesions can occur at successive times and in distant parts of the genome constituting a permanent threat to the genetic integrity. Numerous repair pathways have evolved to reestablish and maintain the genetic information $[1,2]$. The recent identification of DNA methyltransferase I at repair sites indicated that not only the genetic but also the epigenetic information is restored [3].
The repair of DNA lesions involves multiple steps including initial damage recognition, intracellular signaling and the recruitment of repair factors. For the latter step so called loading platforms are considered to play a central role by locally concentrating and coordinating repair factors at sites of DNA damage. These loading platforms have no enzymatic activity of their own but interact with numerous proteins through highly conserved binding motifs. XRCC1 (X-ray cross complementing factor 1) and PCNA (proliferating cell nuclear antigen) both fulfill these criteria and are therefore considered to act as central loading platforms in DNA replication and repair (reviewed in [4-6]). 
XRCC1 was first identified in the Chinese Hamster ovary (CHO) mutant cell line EM9 [7]. This cell line shows a defect in single strand break repair (SSBR) and increased sensitivity to alkylating agents and ionizing irradiation resulting in elevated frequency of spontaneous chromosome aberrations and deletions. The importance of XRCC1 is further underlined by the embryonic lethality of $X R C C 1^{\%}$ mice [8]. The fact that XRCC1 interacts with various proteins involved in SSBR and base excision repair (BER), including PARP-1, PARP-2 [9-11] Polymerase- $\beta$ $[12,13]$ and DNA Ligase III $[9,14]$ suggests that XRCC1 acts as a loading platform in these pathways. Interestingly, XRCC1 also interacts with PCNA and it was proposed that this interaction facilitates efficient SSBR during DNA replication [15].

PCNA forms a homotrimeric ring around the DNA allowing both stable association with and sliding along the DNA double helix. Therefore PCNA is often referred to as a "sliding clamp" mediating interaction of various proteins with DNA in a sequence-independent manner. Photobleaching experiments have shown that in DNA replication PCNA acts as stationary loading platform for transiently interacting Okazaki fragment maturation proteins $[16,17]$. In the last few years it has become evident that PCNA is not only essential for DNA replication but also for various DNA repair pathways including nucleotide excision repair (NER) [18], base excision repair (BER) [19,20], mismatch repair (MMR) [21-23] and repair of double strand breaks (DSBs) $[24,25]$. Recently it has been shown, that accumulation of PCNA at DNA repair sites is independent of the RFC complex, which loads PCNA onto DNA during DNA replication [26]. Furthermore PCNA plays also an important role in postreplicative processes such as cytosine methylation and chromatin assembly $[27,28]$. In most cases, proteins involved in these processes directly bind to PCNA through a conserved PCNA-binding domain (PBD). This raises the question of how binding is coordinated and sterical hindrance avoided in DNA replication and repair. Recent studies have shown that posttranslational modifications of PCNA such as ubiquitinylation and sumoylation [2934] mediate a switch between DNA replication and different repair pathways.

To study the dynamics of the two loading platforms XRCC1 and PCNA at DNA repair sites in Hela cells we used a combination of microirradiation, live cell microscopy and photobleaching techniques. We found that XRCC1 and PCNA exhibit distinct recruitment and binding kinetics at repair sites resulting in different capacities to respond to successive DNA damage events.

\section{Results and discussion $X R C C I$ is less tightly associated with repair sites than PCNA}

XRCC1 and PCNA have no known enzymatic function, are present at repair sites and interact with a high number of different proteins suggesting that they act as loading platforms in DNA repair. To investigate the role of XRCC1 and PCNA in DNA repair we performed immunostainings of microirradiated Hela cells. We employed a confocal laser scanning microscope to generate DNA damage at preselected subnuclear sites with a long wavelength UV diode laser in BrdU-sensitized cells as described before [3,35]. Microirradiated sites stained positive for phosphorylated histone variant $\mathrm{H} 2 \mathrm{AX}(\gamma \mathrm{H} 2 \mathrm{AX})$, a marker for double strand breaks (DSBs), and poly(ADP-Ribose) which is generated by PARP at single strand breaks (SSBs) (Additional file 1). This indicates that microirradiation with a $405 \mathrm{~nm}$ laser generates a mixture of different types of DNA damage that are substrates for distinct DNA repair pathways involving XRCC1 and/or PCNA. Immunofluorescence stainings with specific antibodies revealed that endogenous PCNA and XRCC1 are both present at DNA damage sites as early as 2-4 min after irradiation (Figure 1A). To investigate the binding properties of XRCC1 and PCNA at DNA repair sites we performed salt extraction experiments. Microirradiated cells were permeabilized for $30 \mathrm{~s}$ followed by extraction with phosphate buffer containing $500 \mathrm{mM} \mathrm{NaCl}$ for $1 \mathrm{~min}$. Immediately after salt
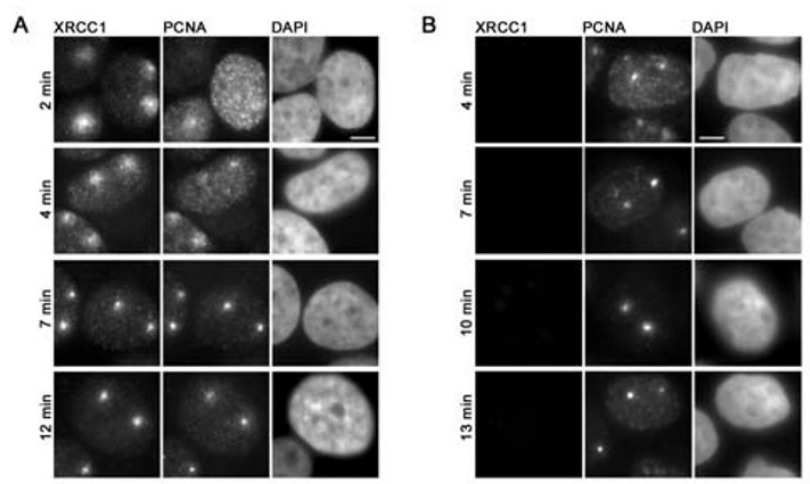

\section{Figure I}

Immunochemical detection of endogenous $\mathrm{XRCCI}$ and PCNA at DNA repair sites. Widefield fluorescence images of Hela cells are shown. Cells were fixed at indicated time points after laser microirradiation. (A) Both, XRCCI and PCNA, accumulate at laser-induced DNA damage sites. (B) Microirradiated Hela cells were extracted with $0,5 \%$ Triton$X I 00$ and $500 \mathrm{mM} \mathrm{NaCl}$ prior to fixation. After in situ extraction no endogenous $\mathrm{XRCCl}$ can be detected at microirradiated sites while PCNA accumulations can still be observed. Scale bars, $5 \mu \mathrm{m}$. 
extraction, the cells were fixed and stained for endogenous proteins showing that XRCC1 and PCNA were both extracted in non-S phase cells that were not microirradiated. In microirradiated non-S phase cells only XRCC1 was extracted while PCNA could still be detected at DNA damage sites (Figure 1B), which is in good agreement with an earlier study, where detergent resistant foci of PCNA could be observed after local UV irradiation [36]. As previously reported [15] we also detected a partial colocalization of XRCC1 with PCNA at replication sites, but noticed dramatically different binding properties. Thus XRCC1 was readily extracted, whereas PCNA was still stably associated with sites of DNA replication (Figure 1B). Taken together these results show that endogenous XRCC1 and PCNA are both present at DNA replication and repair sites but exhibit different binding properties.

\section{Recruitment and mobility of XRCCI and PCNA at DNA repair sites}

To further investigate the dynamics detected with salt extraction experiments we combined the microirradiation technique with live cell microscopy and photobleaching analysis (FRAP). We first determined the recruitment kinetics of XRCC1 and PCNA in living cells by quantifying the amount of GFP- and RFP-tagged XRCC 1 and PCNA accumulated at microirradiated sites. The intensity values were corrected for background and for total nuclear loss of fluorescence over the time course and normalized to the pre-irradiation value.

A direct comparison of GFP- and RFP-tagged XRCC1 and PCNA showed a significantly slower recruitment of PCNA in contrast to the very fast accumulation of XRCC1 at microirradiated sites (Figure 2A). The fluorescence intensity of PCNA at the irradiated site increased during the observation period of $5 \mathrm{~min}$, while XRCC1 accumulation reached a maximum about 1-2 min after irradiation (Figure $2 \mathrm{~B}$ ). These kinetic differences are in good agreement with earlier studies comparing the recruitment of XRCC1 and PCNA to laser-induced DNA damage sites [37].

Having shown that XRCC1 and PCNA are recruited with distinct kinetics we performed FRAP analysis to determine their dynamics at laser-induced DNA damage sites. Two separate spots were microirradiated in living cells coexpressing GFP-XRCC1 and RFP-PCNA. After 5 min one region was bleached with a high energy laser pulse for 300 $\mathrm{ms}$ and the fluorescence recovery was determined. After bleaching of the repair foci we observed complete recovery of the XRCC1 signal within $24 \mathrm{~s}$ (Figure 3 ). Since fluorescence intensity at repair sites had already peaked and did not increase any further, the measured recovery has to be attributed to a rapid turnover of XRCC1.
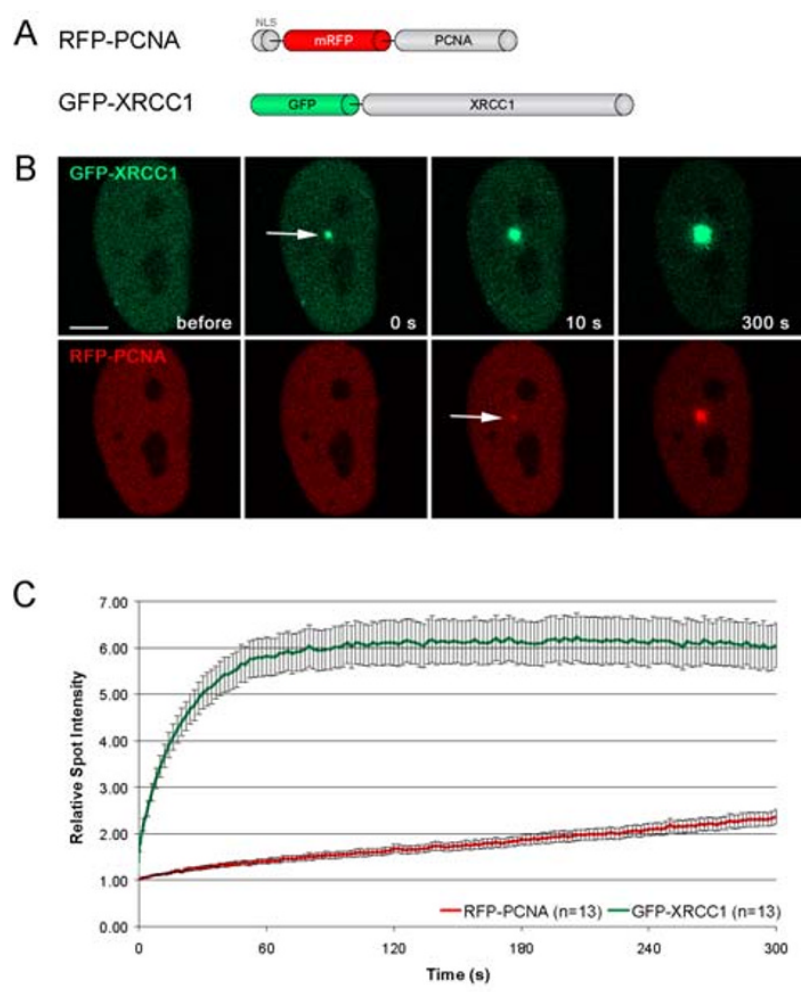

\section{Figure 2}

Recruitment of XRCCI and PCNA at DNA damage sites in living cells. (A) Schematic representation of the fluorescent fusion proteins. (B) Live cell imaging of a microirradiated Hela cell coexpressing GFP-XRCCI and RFP-PCNA. Accumulation of GFP-XRCCI can be observed immediately after microirradiation, while RFP-PCNA accumulates with a short delay of about 2-10 s (indicated by arrows). (C) Quantitative evaluation of recruitment kinetics showing mean curves. Error bars represent the standard error of the mean. Immediate and fast recruitment of GFP-XRCCI precedes slow and constant recruitment of RFP-PCNA at DNA damage sites. Scale bar, $5 \mu \mathrm{m}$.

In contrast, no recovery of PCNA at DNA repair sites could be observed within the observation period, which is in good agreement with previous studies where DNA damage was induced by chemical agents or irradiation with a UV lamp [30,38].

To determine the dissociation kinetics of XRCC1 and PCNA from DNA damage sites we performed FLIP experiments in Hela cells expressing GFP-XRCC1 and RFPPCNA. 5 min after microirradiation half of the nucleus was repeatedly bleached with a high energy laser pulse over a time period of $150 \mathrm{~s}$ and the loss of fluorescence at the microirradiated site located outside the bleaching area was determined (Figure 4, inset). The intensity values 


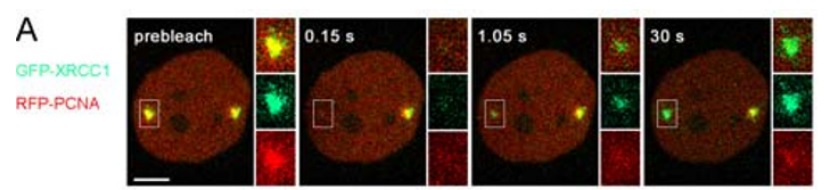

B

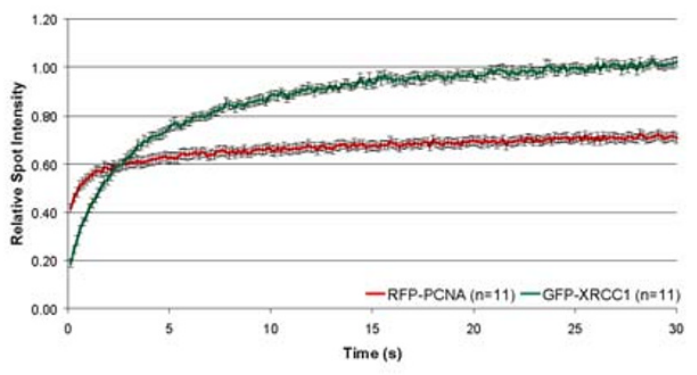

\section{Figure 3}

Mobility of XRCCI and PCNA at DNA damage sites. (A) Two separate subnuclear spots of a transiently transfected Hela cell were microirradiated. The mobility of accumulated fluorescent fusion proteins was determined by bleaching one of the two spots 5 min after microirradiation and subsequent recovery measurements. Inset shows the bleached microirradiated site. Scale bar, $5 \mu \mathrm{m}$. (B) FRAP data from II individual experiments are shown as mean curves. Error bars represent the standard error of the mean.

were corrected for background fluorescence and normalized to the pre-bleach value.

Within the first $10-15 \mathrm{~s}$ both fusion proteins showed a rapid loss of fluorescence due to depletion of highly mobile, unbound fluorescent molecules within the region of interest. After this initial phase XRCC1 and PCNA exhibited dramatically different dissociation kinetics. We could observe a rapid decrease of XRCC1 fluorescence to $10 \%$ of the initial intensity within the observation period while the intensity of PCNA was only reduced to $34 \%$ (Figure 4). This argues for a constant exchange of fluorescent XRCC1 molecules between the damage site and the bleached half of the nucleus, while most RFP-PCNA molecules remained bound at DNA repair sites.

These results show that the two loading platforms XRCC1 and PCNA exhibit distinct recruitment kinetics and mobility (association and dissociation rates) at DNA repair sites, which is consistent with an involvement of XRCC1 and PCNA in distinct repair pathways. On the one hand, PCNA is involved in repair pathways where the synthesis of long stretches of DNA requires a stable and processive repair machinery. On the other hand, XRCC1 is part of the short patch BER pathway where only a single

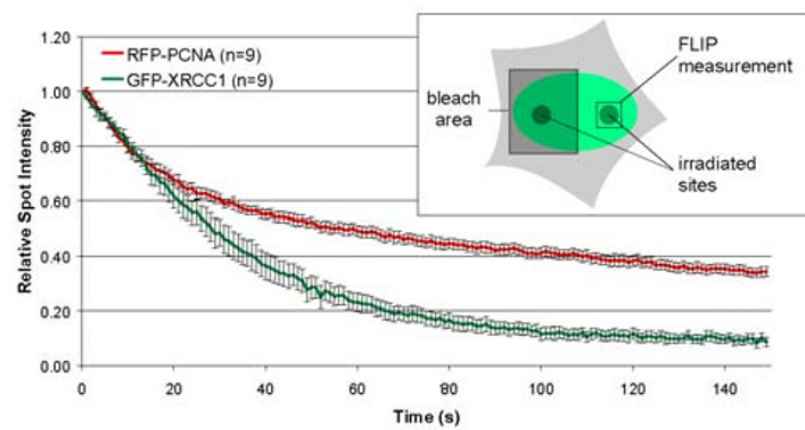

Figure 4

Different binding kinetics of XRCCI and PCNA at DNA repair sites. FLIP data from 9 individual experiments are shown as mean curves. The scheme of the experiment is outlined in the inset. Two separate subnuclear spots of transiently transfected Hela cells were microirradiated. Half of the nucleus containing one irradiated site was repeatedly bleached for I s over a total time period of I50 s, starting 5 min after microirradiation. The decay of the fluorescence intensity at the microirradiated site within the non-bleached half of the nucleus was measured and plotted over time. Error bars represent the standard error of the mean.

nucleotide needs to be replaced and no processive and stable machinery is required.

To further investigate the role of XRCC1 and PCNA as central loading platforms in DNA repair we extended our photobleaching analysis to their respective interaction partners DNA Ligase III and I. In a previous study we compared the recruitment kinetics of theses highly conserved DNA Ligases and found that they are recruited to DNA repair sites with distinct kinetics. Using mutational analysis and binding studies we could show, that DNA Ligase I is recruited to repair sites through interaction with PCNA, while DNA Ligase III is recruited via its BRCT domain interacting with XRCC1 [35]. FRAP analysis revealed that both DNA Ligases show a high turnover at repair sites, with DNA Ligase I recovering faster than DNA Ligase III (Additional file 2). Interestingly, DNA Ligase III showed the same recovery rate as its loading platform XRCC1, while the mobility of DNA Ligase I and PCNA at repair sites differed dramatically (Additional file 2).

These results demonstrate that these loading platforms and their interacting repair factors have independent binding properties at repair sites. We speculate that even transient interaction of repair factors with their respective loading platform enhances the efficiency of DNA repair by local concentration of enzyme activities at repair sites, 
allowing faster recognition and binding of repair substrates.

\section{Flexible response of XRCCI and PCNA to multiple DNA damage events}

To investigate whether the different binding properties of XRCC1 and PCNA have functional consequences we tested their ability to respond to multiple DNA lesions. Successive DNA lesions were introduced with a time interval of $2.5 \mathrm{~min}$ at separate spots and the recruitment kinetics were determined for each individual spot. We observed a constant decrease of PCNA accumulation at sites irradiated at later time points (Figure 5). In contrast, XRCC1 accumulation at early and late irradiated sites was similar.

These differences can be explained by the tight binding of PCNA at repair sites leading to a depletion of the cellular pool of PCNA molecules available for repair of subsequent damages.

In contrast, the dynamic binding of XRCC1 enables fast exchange between multiple DNA damages sites separated in time and space. Taken together these findings argue for a role of PCNA as a stationary loading platform in DNA repair allowing efficient and accurate repair, whereas the fast recruitment and high turnover of XRCC1 enables a flexible response to multiple DNA damage events occurring at distant sites and successive times in the genome.

\section{Conclusion}

In summary, we found that XRCC 1 and PCNA exhibit distinct recruitment and binding kinetics at repair sites, which goes beyond earlier studies comparing only the accumulation of XRCC1 and PCNA at repair sites [37]. Efficient repair of DNA lesions requires avid recognition of the damage and coordinated recruitment of a multitude of repair factors. The principle dilemma faced by the repair machinery is that the stable complex formation required for processivity and completion of multi-step processes is incompatible with a flexible response to later changes like subsequent DNA damages. Our live cell recruitment and photobleaching analyses showed that XRCC1 and PCNA represent opposite strategies. We clearly demonstrate that the stable binding of the processivity factor PCNA limits its capacity to respond to successive damage events. While the avid and transient binding of XRCC1 might be sufficient for single nucleotide replacement but allows a flexible response to multiple consecutive DNA lesions. This type of live cell analysis should also help to explore the flexibility of other repair factors and complex cellular machineries in response to changing requirements.
A

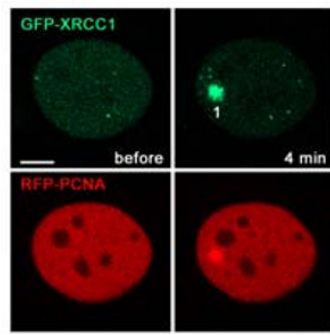

B

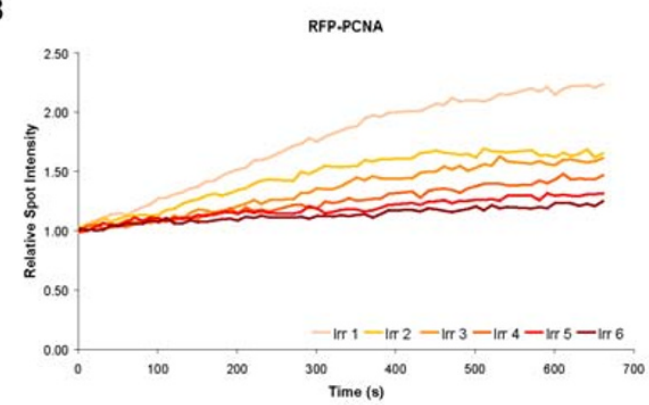

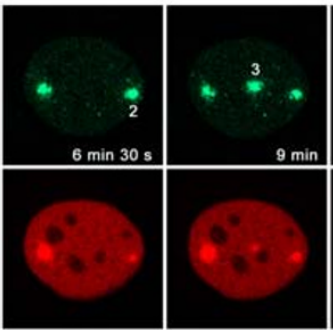
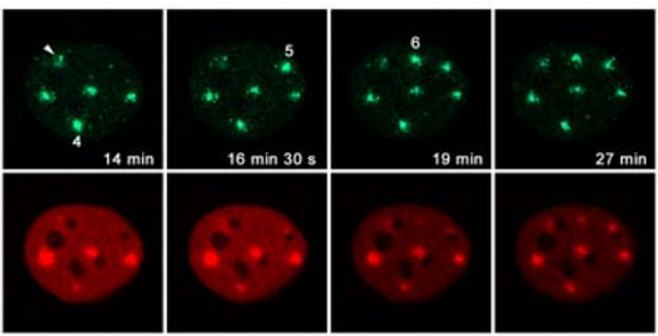

C

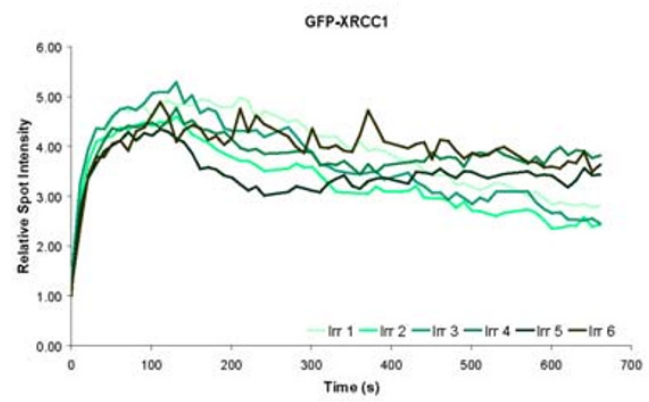

\section{Figure 5}

Flexible response of $\mathrm{XRCCI}$ to multiple DNA damage events. (A) Consecutive/Successive DNA lesions were introduced with a time interval of $2.5 \mathrm{~min}$, starting $4 \mathrm{~min}$ after microirradiation of the first spot. One spot irradiated in close proximity to the nucleoli was not evaluated (arrowhead) (B) The recruitment kinetics of XRCCI and PCNA at consecutively microirradiated sites were evaluated and plotted over time. Representative curves of one Hela cell are shown. 


\section{Methods \\ Cell culture and transfection}

HeLa cells were cultured in DMEM containing $50 \mu \mathrm{g} / \mathrm{ml}$ gentamicin supplemented with $10 \%$ FCS. Cells grown on $\mu$-slides (Ibidi) or on gridded coverslips were cotransfected with jetPEI (PolyPlus Transfection) or TransFectin transfection reagent (Bio-Rad) according to the manufacturers instructions. For microirradiation experiments cells were sensitized by incubation in medium containing BrdU $(10 \mu \mathrm{g} / \mathrm{ml})$ for $24-48 \mathrm{~h}$.

\section{Expression plasmids}

Mammalian expression constructs encoding translational fusions of human PCNA with either green (GFP) or red (RFP) fluorescent protein were previously described [17]. Red variants of the previously described GFP-Ligase III [3] and GFP-XRCC1 [39] were generated by replacing GFP with RFP [40] and termed RFP-Ligase III and RFP-XRCC1, respectively. In all cases expression was under the control of the CMV promoter. We tested all fusion proteins by expression in $293 \mathrm{~T}$ cells followed by western blot analysis.

\section{Immunofluorescence and Detergent Extraction}

Cells were fixed in 3,7\% formaldehyde for $10 \mathrm{~min}$ and permeabilized with ice-cold methanol for $5 \mathrm{~min}$. The following primary antibodies (diluted in PBS containing 2\% BSA) were used: anti- $\gamma$ H2AX (Ser139) rabbit antibody (Upstate), anti-PAR mouse monoclonal antibody (Trevigen), anti-XRCC1 mouse monoclonal antibody (Abcam) and anti-PCNA rat monoclonal antibody [41]. Secondary antibodies (diluted 1:400 in PBS containing 2\% BSA) conjugated to Alexa Fluor 488, 555 or 647 (Molecular Probes) were used. Cells were counterstained with DAPI and mounted in Vectashield (Vector Laboratories). For in situ detergent extraction, cells were permeabilized for $30 \mathrm{~s}$ with $0,5 \%$ Triton X-100 in PBS and extracted for $1 \mathrm{~min}$ with $500 \mathrm{mM} \mathrm{NaCl}$ in PBS before fixation.

\section{Live-cell Microscopy, microirradiation and photobleaching experiments}

Live cell imaging, mircorirradiation and photobleaching experiments were carried out with a Leica TCS SP2/AOBS confocal laser scanning microscope equipped with a UVtransmitting HCX PL $63 \times / 1.4$ oil objective. Fluorophores were exited using a $488 \mathrm{~nm}$ Ar laser line and a $561 \mathrm{~nm}$ diode laser line. The microscope was equipped with a heated environmental chamber set to $37^{\circ} \mathrm{C}$. Confocal image series were typically recorded with a frame size of $256 \times 256$ pixels and a pixel size of $90 \mathrm{~nm}$.

Microirradiation was carried out with a $405 \mathrm{~nm}$ diode laser set to maximum power at $100 \%$ transmission. Preselected spots of $\sim 1 \mu \mathrm{m}$ in diameter within the nucleus were microirradiated for $1 \mathrm{~s}$. Before and after microirradiation confocal image series of one mid z-section were recorded at $2 \mathrm{~s}$ time interval (typically 6 pre-irradiation and 150 post-irradiation frames). For evaluation of recruitment kinetics, fluorescence intensities at the irradiated region were corrected for background and for total nuclear loss of fluorescence over the time course and normalized to the pre-irradiation value.

For FRAP analysis, a region of interest was selected and photobleached for $300 \mathrm{~ms}$ with all laser lines of the Arlaser and the $561 \mathrm{~nm}$ DPSS laser set to maximum power at $100 \%$ transmission. Before and after bleaching, confocal image series were recorded at $150 \mathrm{~ms}$ time intervals (typically 10 prebleach and 200 postbleach frames). Mean fluorescence intensities of the bleached region were corrected for background and for total nuclear loss of fluorescence over the time course and normalized to the mean of the last 4 prebleach values.

For FLIP analysis, one half of the nucleus was repeatedly photobleached (typically 150 frames) with all laser lines of the Ar-laser and the $561 \mathrm{~nm}$ DPSS laser set to maximum power at $100 \%$ transmission for $1 \mathrm{~s}$. Mean fluorescence intensities of the bleached region were corrected for background and normalized to the initial value.

For quantitative evaluation of microirradiation and photobleaching experiments, data of at least 9 nuclei were averaged and the mean curve as well as the standard error of the mean calculated and plotted using Microsoft Excel software.

Images of fixed cells were taken with a Zeiss Axiophot 2 widefield epifluorescence microscope using a Zeiss PlanApochromat $63 \times / 1.40$ oil objective and a cooled CCD camera (Visitron Systems).

\section{Abbreviations}

BER: base excision repair

DSBs: double strand breaks

FLIP: fluorescence loss in photobleaching

FRAP: fluorescence recovery after photobleaching

PCNA: proliferating cell nuclear antigen

SSBR: single strand break repair

SSBs: single strand breaks

XRCC1: X-ray cross complementing factor 1 


\section{Competing interests}

The author(s) declares that there are no competing interests.

\section{Authors' contributions}

OM designed and performed the experiments, analyzed the data and participated in writing the manuscript. HL participated in experimental design and writing the manuscript. All authors read and approved the final manuscript.

\section{Additional material}

\section{Additional file 1}

Laser microirradiation generates different types of DNA damage. Description: The data provided shows that laser microirradiation with a $405 \mathrm{~nm}$ laser generates different types of DNA damage, including SSBs and DSBs. Click here for file

[http://www.biomedcentral.com/content/supplementary/14712199-8-81-S1.doc]

\section{Additional file 2}

Mobility of XRCC1 and PCNA and their respective binding partners DNA Ligase III and I at DNA damage sites. Description: The data provided indicates that XRCC1 and its binding partner DNA Ligase III show similar turnover rates at DNA damage sites, while the mobility of PCNA and its binding partner DNA Ligase I differ dramatically.

Click here for file

[http://www.biomedcentral.com/content/supplementary/14712199-8-81-S2.doc]

\section{Acknowledgements}

We are indebted to Dr R. Tsien for providing the mRFPI expression vector and to Dr. G. de Murcia for providing the GFP-XRCCI expression vector. We are grateful to Drs. M. Cristina Cardoso, Fabio Spada and Gilbert de Murcia for their help and comments on the manuscript. This work was supported by grants from the Deutsche Forschungsgemeinschaft and the Volkswagenstiftung to H.L.

\section{References}

I. Hoeijmakers JH: Genome maintenance mechanisms for preventing cancer. Nature 200 I, 4I I :366-374.

2. Friedberg EC: DNA damage and repair. Nature 2003, 421:436-440.

3. Mortusewicz O, Schermelleh L, Walter J, Cardoso MC, Leonhardt $\mathrm{H}$ : Recruitment of DNA methyltransferase I to DNA repair sites. Proc Natl Acad Sci U S A 2005.

4. Warbrick E: The puzzle of PCNA's many partners. Bioessays 2000, 22:997-1006.

5. Caldecott KW: XRCCI and DNA strand break repair. DNA Repair (Amst) 2003, 2:955-969.

6. Maga G, Hubscher U: Proliferating cell nuclear antigen (PCNA): a dancer with many partners. J Cell Sci 2003, I | 6:305|-3060.

7. Thompson LH, Brookman KW, Dillehay LE, Carrano AV, Mazrimas JA, Mooney CL, Minkler JL: A CHO-cell strain having hypersensitivity to mutagens, a defect in DNA strand-break repair, and an extraordinary baseline frequency of sister-chromatid exchange. Mutat Res 1982, 95:427-440.

8. Tebbs RS, Flannery ML, Meneses J], Hartmann A, Tucker JD, Thompson LH, Cleaver JE, Pedersen RA: Requirement for the Xrcc
DNA base excision repair gene during early mouse development. Dev Biol 1999, 208:513-529.

9. Caldecott KW, McKeown CK, Tucker JD, Ljungquist S, Thompson $\mathrm{LH}$ : An interaction between the mammalian DNA repair protein XRCCI and DNA ligase III. Mol Cell Biol I994, I 4:68-76.

10. Masson M, Niedergang C, Schreiber V, Muller S, Menissier-de Murcia $J$, de Murcia $G$ : XRCCI is specifically associated with poly(ADP-ribose) polymerase and negatively regulates its activity following DNA damage. Mol Cell Biol 1998, | 8:3563-357|.

II. Schreiber V, Ame JC, Dolle P, Schultz I, Rinaldi B, Fraulob V, Menissier-de Murcia J, de Murcia G: Poly(ADP-ribose) polymerase-2 (PARP-2) is required for efficient base excision DNA repair in association with PARP-I and XRCCI. J Biol Chem 2002, 277:23028-23036.

12. Kubota Y, Nash RA, Klungland A, Schar P, Barnes DE, Lindahl T: Reconstitution of DNA base excision-repair with purified human proteins: interaction between DNA polymerase beta and the XRCCI protein. Embo J 1996, I 5:6662-6670.

13. Caldecott KW, Aoufouchi S, Johnson P, Shall S: XRCCI polypeptide interacts with DNA polymerase beta and possibly poly (ADP-ribose) polymerase, and DNA ligase III is a novel molecular 'nick-sensor' in vitro. Nucleic Acids Res 1996, 24:4387-4394.

14. Wei YF, Robins P, Carter K, Caldecott K, Pappin DJ, Yu GL, Wang RP, Shell BK, Nash RA, Schar P, et al.: Molecular cloning and expression of human cDNAs encoding a novel DNA ligase IV and DNA ligase III, an enzyme active in DNA repair and recombination. Mol Cell Biol 1995, I 5:3206-32 I6.

15. Fan J, Otterlei M, Wong HK, Tomkinson AE, Wilson DM 3rd: XRCCI co-localizes and physically interacts with PCNA. Nucleic Acids Res 2004, 32:2193-2201.

16. Sporbert A, Gahl A, Ankerhold R, Leonhardt H, Cardoso MC: DNA polymerase clamp shows little turnover at established replication sites but sequential de novo assembly at adjacent origin clusters. Mol Cell 2002, I0:1355-1365.

17. Sporbert A, Domaing P, Leonhardt H, Cardoso MC: PCNA acts as a stationary loading platform for transiently interacting Okazaki fragment maturation proteins. Nucleic Acids Res 2005 , 33:3521-3528.

18. Shivji KK, Kenny MK, Wood RD: Proliferating cell nuclear antigen is required for DNA excision repair. Cell 1992, 69:367-374

19. Gary R, Kim K, Cornelius HL, Park MS, Matsumoto Y: Proliferating cell nuclear antigen facilitates excision in long-patch base excision repair. J Biol Chem 1999, 274:4354-4363.

20. Levin DS, McKenna AE, Motycka TA, Matsumoto Y, Tomkinson AE: Interaction between PCNA and DNA ligase $I$ is critical for joining of Okazaki fragments and long-patch base-excision repair. Curr Biol 2000, 10:919-922.

21. Umar A, Buermeyer AB, Simon JA, Thomas DC, Clark AB, Liskay RM, Kunkel TA: Requirement for PCNA in DNA mismatch repair at a step preceding DNA resynthesis. Cell I996, 87:65-73.

22. Johnson RE, Kovvali GK, Guzder SN, Amin NS, Holm C, Habraken Y, Sung P, Prakash L, Prakash S: Evidence for involvement of yeast proliferating cell nuclear antigen in DNA mismatch repair. Biol Chem 1996, 27 I:27987-27990.

23. Jiricny J: The multifaceted mismatch-repair system. Nat Rev Mol Cell Biol 2006, 7:335-346.

24. Holmes AM, Haber JE: Double-strand break repair in yeast requires both leading and lagging strand DNA polymerases. Cell 1999, 96:415-424.

25. Dorazi R, Parker JL, White MF: PCNA activates the Holliday junction endonuclease Hjc. J Mol Biol 2006, 364:243-247.

26. Hashiguchi K, Matsumoto $Y$, Yasui A: Recruitment of DNA repair synthesis machinery to sites of DNA damage/repair in living human cells. Nucleic Acids Res 2007.

27. Moggs JG, Grandi P, Quivy JP, Jonsson ZO, Hubscher U, Becker PB Almouzni G: A CAF-I-PCNA-mediated chromatin assembly pathway triggered by sensing DNA damage. Mol Cell Biol 2000 , 20: $1206-1218$.

28. Chuang LS, lan HI, Koh TW, Ng HH, Xu G, Li BF: Human DNA(cytosine-5) methyltransferase-PCNA complex as a target for p2 IWAFI. Science 1997, 277:|996-2000.

29. Pfander B, Moldovan GL, Sacher M, Hoege C, Jentsch S: SUMOmodified PCNA recruits Srs 2 to prevent recombination during S phase. Nature 2005, 436:428-433. 
30. Solomon DA, Cardoso MC, Knudsen ES: Dynamic targeting of the replication machinery to sites of DNA damage. J Cell Biol 2004, 166:455-463.

3I. Hoege C, Pfander B, Moldovan GL, Pyrowolakis G, Jentsch S: RAD6dependent DNA repair is linked to modification of PCNA by ubiquitin and SUMO. Nature 2002, 4I 9: |35- I4I.

32. Matunis MJ: On the road to repair: PCNA encounters SUMO and ubiquitin modifications. Mol Cell 2002, 10:44I-442.

33. Stelter P, Ulrich HD: Control of spontaneous and damageinduced mutagenesis by SUMO and ubiquitin conjugation. Nature 2003, 425: I88-191.

34. Papouli E, Chen S, Davies AA, Huttner D, Krejci L, Sung P, Ulrich HD: Crosstalk between SUMO and ubiquitin on PCNA is mediated by recruitment of the helicase Srs2p. Mol Cell 2005, 19:123-133.

35. Mortusewicz O, Rothbauer U, Cardoso MC, Leonhardt H: Differential recruitment of DNA Ligase I and III to DNA repair sites. Nucleic Acids Res 2006, 34:3523-3532

36. Okano S, Lan L, Caldecott KW, Mori T, Yasui A: Spatial and temporal cellular responses to single-strand breaks in human cells. Mol Cell Biol 2003, 23:3974-398I.

37. Lan L, Nakajima S, Oohata Y, Takao M, Okano S, Masutani M, Wilson $\mathrm{SH}$, Yasui A: In situ analysis of repair processes for oxidative DNA damage in mammalian cells. Proc Natl Acad Sci U S A 2004 I 01: 13738-13743.

38. Essers J, Theil AF, Baldeyron C, van Cappellen WA, Houtsmuller AB, Kanaar R, Vermeulen W: Nuclear dynamics of PCNA in DNA replication and repair. Mol Cell Biol 2005, 25:9350-9359.

39. Levy N, Martz A, Bresson A, Spenlehauer C, de Murcia G, Menissierde Murcia J: XRCCI is phosphorylated by DNA-dependent protein kinase in response to DNA damage. Nucleic Acids Res 2006, 34:32-4I.

40. Campbell RE, Tour O, Palmer AE, Steinbach PA, Baird GS, Zacharias DA, Tsien RY: A monomeric red fluorescent protein. Proc Natl Acad Sci U S A 2002, 99:7877-7882.

41. Spada F, Haemmer A, Kuch D, Rothbauer U, Schermelleh L, Kremmer E, Carell T, Langst G, Leonhardt H: DNMTI but not its interaction with the replication machinery is required for maintenance of DNA methylation in human cells. J Cell Biol 2007, I 76:565-57|.

Publish with Bio Med Central and every scientist can read your work free of charge

"BioMed Central will be the most significant development for disseminating the results of biomedical research in our lifetime. "

Sir Paul Nurse, Cancer Research UK

Your research papers will be:

- available free of charge to the entire biomedical community

- peer reviewed and published immediately upon acceptance

- cited in PubMed and archived on PubMed Central

- yours - you keep the copyright
BiolMedcentral 